\title{
Social-sine cosine algorithm-based cross layer resource allocation in wireless network
}

\author{
Praveena T., Nagaraja G. S.
}

Department of Computer Science and Engineering, RV College of Engineering, India

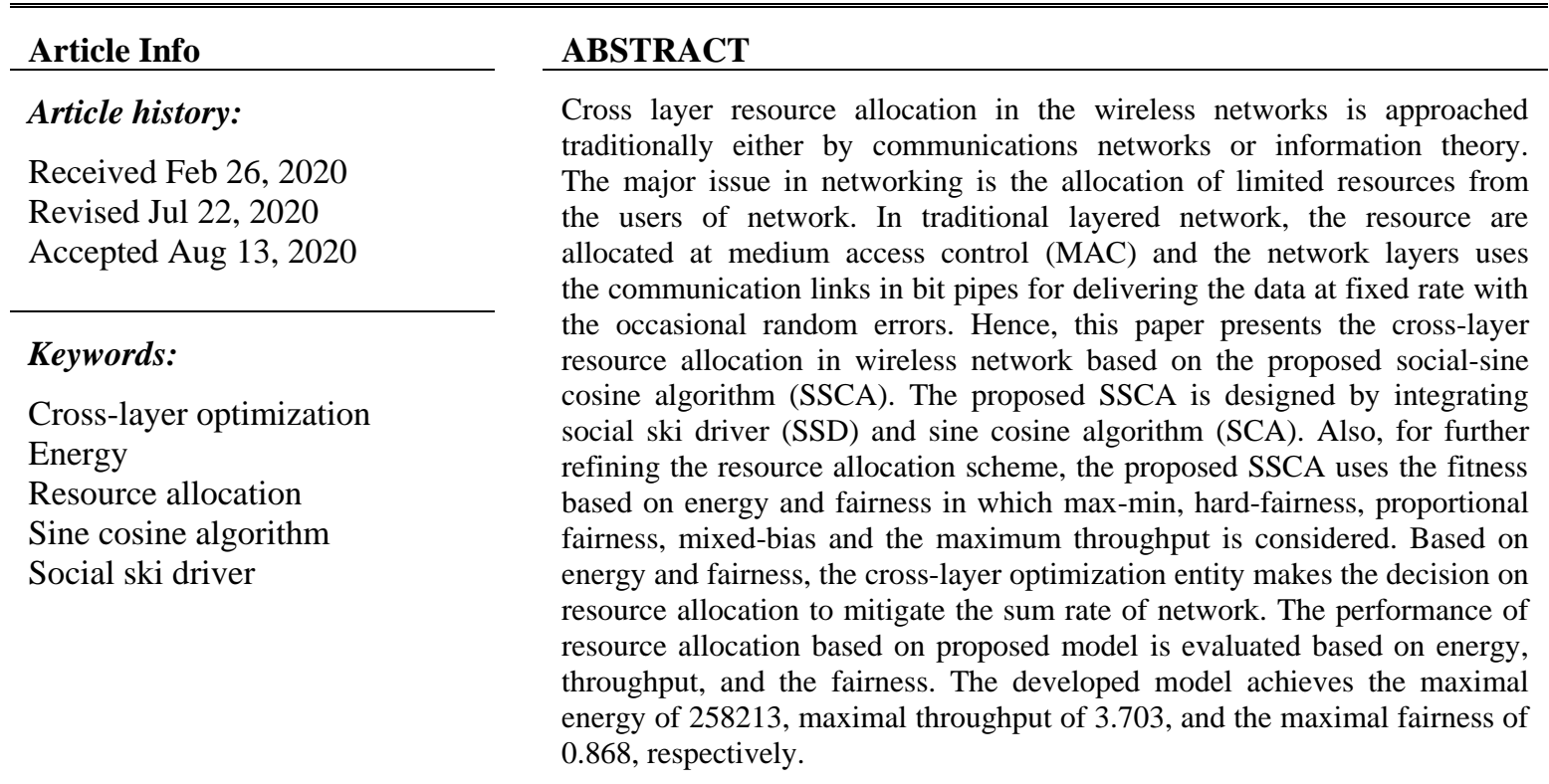

This is an open access article under the CC BY-SA license.

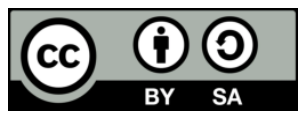

\section{Corresponding Author:}

Praveena T.,

Department of Computer Science and Engineering,

RV College of Engineering,

Bengaluru, Karnataka, India.

Email: praveenat@rvce.edu.in

\section{INTRODUCTION}

In the last few decades, the wireless communications have been grown rapidly in the worldwide. However, the upcoming wireless communication has provided various services of high-quality voice to the wireless multimedia for everywhere at low cost [1]. The wireless network is also said to be International Mobile Telecommunications 2000 (IMT-2000) for providing the services using multimedia mobile to attain the high bit rate of about $2 \mathrm{Mb} / \mathrm{s}$ [2]. To fulfill user requirements, the service provider includes various techniques, like cross-layer design and co-operative relaying in the wireless communication systems. The co-operative relaying is utilized to improve the coverage of base station (BS) and the system capacity in wireless communication network [3, 4]. While varying the channel-state information (CSI) and queue-state information (QSI) in wireless network, the nodes accept their reception and the transmission parameters with power constraints and the quality of service (QoS) needs [5], but still the channels undergo time varying multipath fading. Additionally, the inflexibility and the sub-optimality results in inefficient resource in the wireless networks $[6,7]$. 
The resource management and the allocation are very difficult for the wireless networks where the spectral resources are provided by several users. In the layered networking structure, every layer is operated as well as designed separately [6]. Some of the resource allocation schemes employed for mobile communication systems are service level agreements and the QoS parameters. Here, the QoS parameters containing objective measures, like transfer delay, packet loss ratio, guaranteed bit rate, delay variation and so on. Thus, the improved subjective quality perceptron is very significant to the providers for maintaining and increasing the customer needs. However, the resource allocation includes algorithms and strategies in order to control the parameters, like channel allocation, transmit power, modulation scheme, data rates, error coding scheme and so on. In general, the scheduling approaches use the number of hops, transmission distance, delay, channel condition [8].

Cross-layer optimization is broadly utilized for providing QoS provisioning in wireless multimedia communication $[9,10]$ in the wireless multimedia communications $[9,10]$. The cross-layer scheme works with conventional network model to improve the system performance, but the design principle may cause high complexity and several optimization issues $[9,11]$. Accordingly, the efficient cross-layer techniques are introduced to reduce the additional requested information from both the practical and the theoretical point of view [9]. Currently, the cross-layer architecture is designed to improve the system performance in modern wireless networks, and in addition, the cross-layer interaction patterns are supported with protocol stack beyond 3G mobile communication systems [12-13]. In [14], the cross-layer resource allocation was established by considering both the data link layer and the physical layer.

This paper presents an approach for cross layer resource allocation based on SSCA. Here, the crosslayer design shares the information through layer boundaries for determining the data receives from the other layers. Hence, the cross-layer design is utilized for sharing the status, parameters, and the other information to other four layers. By changing the channel conditions, the cross-layer optimization entity updates the decision on the basis of new input data. In this case, the resource allocation strategy is performed based on proposed SSCA, which is designed newly by combining SSD and SSA. The fitness function for best resource allocation is evaluated on the basis of energy and fairness. The fairness parameters, like max-min, hard-fairness, maximum throughput, proportional fairness, and maximum throughput are considered.

The major contribution of the paper is:

- Proposed SSCA-based resource allocation: The cross-layer resource allocation framework named SSCA-based allocation is proposed for allocating the resources effectively. The location of search agents is updated using the fitness function by considering fairness and energy.

The paper is arranged according to following series: section 2 elaborates existing methods of resource allocation with challenges of methods that remain as motivation for research. The system model of resource allocation is portrayed in section 3 and the developed method of resource allocation is explained in section 4. The results of the methods are deliberated in section 5. At last, section 6 concludes the work.

\section{MOTIVATION}

This section presents the literature survey of several methods utilized for resource allocation and the challenges of the existing works are discussed.

\subsection{Literature survey}

Several methods related to cross layer resource allocation techniques are described, and analyzed as follows: Maleki and Mirjalily [15] developed comprehensive cross-layer resource allocation approach for providing fairness, robustness and balancing, and the throughput maximization. Here, the power consumption was found better, but other metrics, like max-min and proportional fairness was not considered. Tseng, et al. [16] developed single-input multiple-output (SIMO) that consider the angle between jammer and channel vector in the antenna spatial domain. After that, the new best solution for anti-jamming cross layer resource allocation was derived. Here, peak signal-to-noise ratio (PSNR) was improved, but the method failed to consider other algorithms for better system performance. Jung, et al. [17] presented an approach was introduced for the wireless video transmission. Here, two metrics were considered for measuring relative difference of each forward error correction (FEC) block to quality of user experience. Here, the computational complexity was greatly reduced, but the unequal error protection (UEP) resource allocation are not considered for hybrid automatic repeat request transmission, and multicast video packets. Kordbacheh, et al. [18] employed robust cross layer routing and the radio resource allocation in the wireless ad-hoc networks. In this case, the performance gain was found better, however, the optimization problem is still difficult because of non-convexity.

$\mathrm{Xu}$, et al. [19] presented security-based energy efficient for multi-homing networks. Here, the security-enabled resource allocation was formulated as the maximization issue of dropping probability, 
packet delay, and the availability of power consumption. The method failed to solve the multi-eves problem. Senthilkumar and Meenakshi [3] developed the approach for resource allocation strategy for multidestination relay systems in the cellular downlink. The QoS requirements utilized in this work is minimum data rate requirements, maximum delay threshold, and the zero overflows. However, the method did not analyze both downlink and uplink performance. $\mathrm{Xu}$ and Zhuang [20] presented cross-layer resource allocation for the heterogeneous wireless network. Here, the resource allocation was subjected to service outage probability, constraints in delay, system ratio bandwidth, and the total power consumption. The energy efficiency was increased in this work, but the cross-tier interference was not considered for bandwidth allocation at the heterogeneous wireless access. Tseng, et al. [21] presented an approach for resource allocation crossing the application and physical layers for uplink MU-MIMO OFDMA systems. This approach achieves better improvement in average peak-signal-to-noise-ratio (PSNR), but the user grouping was not analyzed.

\subsection{Challenges}

The challenge that has been identified by analyzing the previous cross layer resource allocation techniques are listed below.

- The challenges faced by cross-layer design is the coexistence problem, which means it is very complex for integrating various cross-layer designs to uniform, because of the specific communication standard of each cross-layer design [13].

- Another challenge faced by cross layer design is overhead caused by cross layer signaling. The crosslayer shared the information among the nodes is very complex and challenging [13].

- Standardization provides the unique vehicle to smooth several cross-layer design solutions in mobile communication networks, but the specification, investigation, development, and the standardization of cross-layer entities failed to meet the requirement of cross-layer optimizations as well as the dynamic interaction patterns between protocol layers remains open challenge [12].

- Unlike single-carrier network, the multi-carrier network serves various users at the particular time; therefore, the multi-carrier scheduling design for the bursty traffic is the hectic challenge [6].

- In [15], Comprehensive cross-layer resource allocation model is devised for fault tolerant topology control in the wireless mesh networks. Here, the method failed to consider the fairness and the energy model together for resource allocation among the multiple layers.

\section{SYSTEM MODEL}

This section presents the system model for cross layer resource allocation in CDMA-based wireless as-hoc network. Let us consider the energy constrained Cognitive Radio orthogonal frequency division multiple access (CR OFDMA) with $M$ communicating pairs. Here, both the transmitter $u$ and the receiver $v$ are represented as $K:=\{1,2, \ldots, n\}$. If $u=v$, then the transmission system is considered as the time slotted OFDMA system at the particular time interval $\tau_{L}$. Here, the slot synchronization is attained using beaconing approach. For every time slot, the particular time interval is given for achieving synchronization to perform spectrum detection and resource allocation. However, inter-carrier interference (ICI) produced by the frequency offset of side lobes pertaining to the transmitter $u$. In the physical layer, the frequency-based Rayleigh fading channel is considered for dividing whole spectrum into $N$ subcarriers for guaranteeing every subcarrier by experiencing Rayleigh fading. The subcarrier set present in transmitter and receiver pair $u$ is denoted as, $\boldsymbol{G}_{u} \subset\{1,2, \ldots, N\}$. Let us consider $\boldsymbol{H}:=\left\{\boldsymbol{H}_{u, v}^{l}, \boldsymbol{u}, v \in \boldsymbol{M}, \boldsymbol{l} \in \boldsymbol{G}_{u}\right\}$, which represents the subcarrier fading co-efficient matrix, where, the term $\boldsymbol{H}_{u, v}^{l}$ denotes the sub-channel coefficient gain from $u$ to $v$ at the subcarrier $l$, and is expressed as,

$$
H_{u, v}^{l}=\left|I_{u, v}^{l}(e)\right|^{2}
$$

where, the term $\left|I_{u, v}^{l}(e)\right|$ refer to the transfer function. Here, the term $H$ uses the block fading channel ofsize $\tau_{L}$ that remains invariant through blocks and the uncorrelated over successive blocks. The noise considered here is additive white gaussian noise (AWGN) with the variance $\sigma_{u, l}^{2}$ at receiver $u$ over subcarrier $l$. Then, the transmission power allocation matrix is indicated as $Q=\left\{q_{u}^{l}, q_{u}^{l} \geq 0 ; u \in K, l \in G_{u}\right\}$ for 
the entire users in $K$ over available subcarrier set $V_{u \in K} G_{u}$, where the term $q_{u}^{l}$ denotes the power allocated to subcarrier at transmitter $u$. For each transmitter, the power vector is given by,

$$
g_{u}:=\left[g_{u}^{1}, g_{u}^{2}, \ldots, g_{u}^{N}\right]^{\tau}
$$

The energy consumption per information rate to achieve high energy efficiency for the transmitter receiver pair at each time slot is denoted by,

$$
E_{u}\left(g_{u}, a_{u}\right):=\frac{\sum_{l \in G_{u}} g_{u}^{l}+g_{u}^{s}}{\sum_{l \in G_{u}} a_{u}^{l}}
$$

where, the term $a_{u}^{l}$ refer to the data rate of $l^{\text {th }}$ subcarrier. The power allocated over $l^{\text {th }}$ subcarrier for $u^{\text {th }}$ transmitter is represented as $g_{u}^{l}$, and the term $g_{u}^{s}$ signifies the receiving power.

\section{PROPOSED RESOURCE ALLOCATION ALGORITHM}

This section illustrates the proposed SSCA algorithm for resource allocation in wireless network. The cross-layer optimization is the combination of joint scheduling and resource allocation in wireless network along with medium access control (MAC), physical layer and the application layer are included in the unified cross-layer optimization. Here, the cross-layer optimization algorithm gets queue state information (QSI) and channel state interference (CSI) from MAC and physical layer. Consequently, the other input parameters, like energy and fairness, the cross-layer optimization entity makes the decision on resource allocation to maximize the sum rate of network. By varying the channel conditions, the cross-layer optimization entity updates the decision based on new input data. Here, the novel resource allocation strategy is developed using the proposed SSCA, which will be newly designed by integrating SSD [22] and SSA [23]. Also, for further refining the resource allocation scheme, the proposed SSCA uses the fitness based on energy [19] and fairness where the hard-fairness, proportional fairness, mixed-bias max-min, and maximum throughput is considered. The block diagram of the proposed SSCA-based resource allocation in the wireless network is shown in Figure 1.

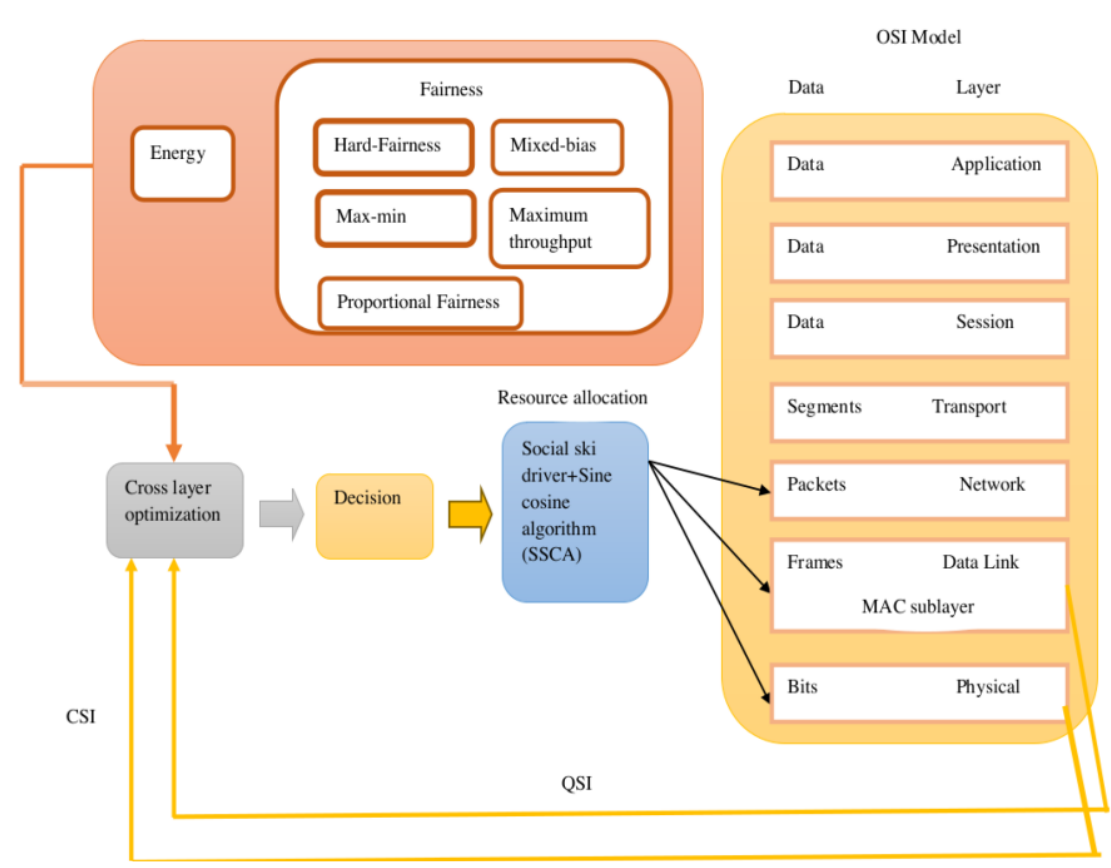

Figure 1. Schematic view of the cross layer resource allocation using the proposed social-sine cosine algorithm 


\subsection{Cross layer optimization}

Cross-layer optimization model consists of different layers and different parameters. The three layers present in the cross-layer optimization. The adaptive modulation and coding (AMC) and CSI-reference signal (CSI-RS) feedback are available in physical layer, whereas the subcarrier assignment, adaptive power control, automatic repeat request (ARQ), forward error correction (FEC), and quality of service in the MAC layer along with the adaptive transmission rate in the network layer. In traditional OSI, the strict boundaries are present among the layers in which the data are provided in the given particular layer and every layer gives the independent solution with the own optimized adaptation, but it is very complex to fulfill all the requirements, like data rate, bit error rate, and the latency for various services. In the dynamic wireless networks, the QSI and CSI vary with the time, hence the network node adapts the reception and the transmission parameters to satisfy the power constraint and the QoS needs [5, 24].

\subsection{Proposed social-sine cosine algorithm for resource allocation}

The proposed SSCA is the combination of SSD with SSA. The SSD algorithm is inspired by various evolutionary optimization approaches to minimize the SVMs parameters with the aim to enhance the system performance. The main aim of SSD is to search in the space for optimal or near-optimal solutions. This method is very efficient for generating improved features to tackle multi-objective optimization issues. Moreover, the method can solve the highly non-linear problems with complex constraints. The SCA algorithm is the population-based optimization and there is no guarantee for finding the solution with single run. This approach is utilized for creating various random solutions and fluctuate them towards the best solution based on sine function. Here, various random and adaptive variables are combined in order to emphasize the exploitation and exploration of the search space. The merits of the algorithm are that the algorithm exhibits better convergence speed, minimal error, and minimal computational time. Hence, integrating SSD in SSA produces better solution with the improved system performance.

The developed SSCA for cross layer resource allocation is illustrated in this section. The proposed SSCA is the combination of SSD [22] with SSA [23], and thus attains the advantages of SSD in SSA. The SSD algorithm is inspired by various evolutionary optimization approaches to minimize the SVMs parameters with the aim to enhance the system performance. The main aim of SSD is to search in the space for optimal or near-optimal solutions. This method is very efficient for generating improved features to tackle multi-objective optimization issues. Moreover, the method can solve the highly non-linear problems with complex constraints. The SCA algorithm is the population-based optimization and there is no guarantee for finding the solution with single run. This approach is utilized for creating various random solutions and fluctuate them towards the best solution based on sine function. Here, various random and adaptive variables are combined in order to emphasize the exploitation and exploration of the search space. The merits of the algorithm are that the algorithm exhibits better convergence speed, minimal error, and minimal computational time. Hence, integrating SSD in SSA produces better solution with the improved system performance. The advantages of the proposed method are better convergence speed, minimal error, minimal computation time and it produces better solution with the improved system performance.

\subsubsection{Solution encoding}

The solution encoding is the representation that is identified with the developed model. Assume $d$ number of transmitters and $m$ number of subcarriers from which $s$ optimal solution is chosen by the developed model such that $s$ value ranging from $1 \leq s \leq b$, respectively. Here, the solution requires for deciding which transmitter to be allocated in which subcarrier.

\subsubsection{Objective modelling}

The fitness function is evaluated in order to obtain the better result. The optimal solution is determined from the previous iteration as each solution to obtain the better location. The objective function of the developed approach is formulated in terms of energy, and fairness, and is expressed by,

$$
f=\sum_{u=1}^{R}\left(\left(1-D_{u}\right)+F_{u}\right)
$$

where, the term $D_{u}$ denotes the transmitter $u$, the fairness is represented as $F_{u}$ and the number of transmitter is indicated as $R$.

a. Fairness for resource allocation: The fairness parameters, like hard-fairness $\boldsymbol{J}_{u}$, max-min fairness $\boldsymbol{P}_{u}$, proportional fairness $B_{u}$, mixed-bias $T_{u}$ and the maximum throughput $X_{u}$ [25] are given below, 
b. Hard-fairness: It is also termed as round robin (RR)-based resource allocation. It is utilized for allocating time or frequency between the potential candidates with respect to any metric. Simple time division multiplexing (TDM) is the best example for RR scheduler where every node is given as the time slot to transmit in the regular intervals. The expression for hard fairness is denoted as,

$$
J_{u}=\frac{A^{s}}{\text { Countof users }}
$$

where, the term $A^{S}$ indicates the maximum number of users who equally shared the resources.

c. Max-min fairness: In Max-min fairness, the less number of resources allocated to every node is increased. In other words, the gap is minimized among maximum and minimum number of assigned resources to each user.

$$
P_{u}=\max _{\left\{g_{b u}^{(l)}\right\}} \min _{u} c_{u}
$$

where, the transmit power of relay $b$ helping the source $u$ on subcarrier $l$ is denoted as $g_{b u}^{(l)}$, and the data rate is represented as $c_{u}$.

d. Proportional fairness: It implements time-enabled fairness and provides the good tradeoff among fairness and network throughput with respect to max-min fairness. Here, the nodes with the lower data rate take more time than those with the higher data rates, which leads to reduced network throughput. The proportional fairness is expressed as,

$$
B_{u}=\frac{1}{\eta^{h}}
$$

where, the term $\eta$ represents the characteristic which priority is assigned to $\eta>0$, and the proportionality factor is indicated as $\boldsymbol{h}, \boldsymbol{h}>\mathbf{O}$.

e. Mixed-bias: Mixed-bias aims to allocate the portion of total capacity available at the node through strongly biased policy, and rests are employed as the fairer policy. The expression of mixed-bias is given by,

$$
T_{u}=\frac{\alpha}{W^{p_{1}}}+\frac{(1-\alpha)}{W^{p_{2}}}
$$

where, the terms $p_{1}$ and $p_{2}$ represents the proportionality factor, $p_{1}, p_{2}>0$ and $\alpha \geq 0$.

f. Maximum throughput: It is concerned only with resources allocation for maximizing the throughput. Here, the node that transmits more data, gets access to resources first to obtain high sum-throughput.

\subsubsection{Algorithmic steps of the developed SSCA-based allocation}

The proposed SSCA is designed by integrating SCA in SSD. Here, the update equation of SSD is modified using the update equation of SCA algorithm. The modification makes the solution update to be more efficient, and it further improves the convergence of the optimization algorithm. The steps followed in the developed algorithm are illustrated below:

Step 1: Initialization: The first step of the proposed SSCA algorithm is the initialization of the position of search agents in which, the total number of agents are identified by the user. The location of agents is represented as,

$$
X_{v}^{t} ;(1 \leq v \leq z)
$$

where, $X_{v}^{t}$ represents the agents position at time $t$, and the number of variables are denoted as $v$.

Step 2: Objective function evaluation: The fitness is calculated for each solution on the basis of fitness function depicted in (4). The fitness function is considered as the maximization function, and solution with maximum fitness is considered as the best solution. 
Step 3: Update the solution based on SSCA algorithm: After evaluating the objective function, the solution undergoes position update on the basis of SSCA. The update equation of SSD velocity $k_{v}^{t}$ is expressed as,

$$
X_{v}^{t+1}=X_{v}^{t}+k_{v}^{t}
$$

when $m_{2} \leq 0.5$

$$
\begin{aligned}
& X_{v}^{t+1}=X_{v}^{t}+e \sin \left(m_{1}\right)\left(\mathrm{B}_{v}^{t}-X_{v}^{t}\right)+\sin \left(m_{1}\right)\left(\mathrm{A}_{v}^{t}-X_{v}^{t}\right) \\
& X_{v}^{t+1}=X_{v}^{t}+e \sin \left(m_{1}\right) \mathrm{B}_{v}^{t}-e \sin \left(m_{1}\right) X_{v}^{t}+\sin \left(m_{1}\right) \mathrm{A}_{v}^{t}-\sin \left(m_{1}\right) X_{v}^{t} \\
& X_{v}^{t+1}=X_{v}^{t}\left(1-e \sin \left(m_{1}\right)-\sin \left(m_{1}\right)\right)+e \sin \left(m_{1}\right) \mathrm{B}_{v}^{t}+\sin \left(m_{1}\right) \mathrm{A}_{v}^{t}
\end{aligned}
$$

where, the term $k_{v}$ denotes the velocity of $X_{v}$, and the uniformly distributed random numbers are indicated as $m_{1}$ and $m_{2}$ ranging from 0 to 1 . The term $\mathrm{B}_{v}$ represents the best solution of $v^{\text {th }}$ agent, and the term $\mathrm{A}_{v}$ indicates the mean global solution for whole population. The above equation shows the update equation of SSD, which is integrated with update equation of SCA. Hence, the update equation of SCA by taking the condition $Y_{4}<0.5$ is expressed by,

$$
X_{v}^{t+1}=X_{v}^{t}+Y_{1} \sin \left(Y_{2}\right) \times\left|Y_{3} \mathbf{I}_{v}^{t}-X_{v}^{t}\right|
$$

assume $I_{v}^{t}>X_{v}^{t}$

$$
\begin{aligned}
& X_{v}^{t+1}=X_{v}^{t}+Y_{1} \sin \left(Y_{2}\right) \times\left(Y_{3} \mathbf{I}_{v}^{t}-X_{v}^{t}\right) \\
& X_{v}^{t+1}=X_{v}^{t}+Y_{1} Y_{3} \sin \left(Y_{2}\right) \mathbf{I}_{v}^{t}-Y_{1} \sin \left(Y_{2}\right) X_{v}^{t} \\
& X_{v}^{t+1}=X_{v}^{t}\left(1-Y_{1} \sin \left(Y_{2}\right)\right)+Y_{1} Y_{3} \sin \left(Y_{2}\right) \mathbf{I}_{v}^{t} \\
& X_{v}^{t}=\frac{1}{1-Y_{1} \sin \left(Y_{2}\right)}\left[X_{v}^{t+1}-Y_{1} Y_{3} \sin \left(Y_{2}\right) \mathbf{I}_{v}^{t}\right]
\end{aligned}
$$

where, the term $X_{v}^{t}$ represents the location of current solution at $t^{\text {th }}$ iteration, $I_{v}^{t}$ refer to the target position, denotes the absolute value, and the random numbers are denoted as $Y_{1}, Y_{2}$, and $Y_{3}$ are the random numbers, respectively. Substituting (18) in (13),

$$
\begin{aligned}
& X_{v}^{t+1}=\frac{X_{v}^{t+1}-Y_{1} Y_{3} \sin \left(Y_{2}\right) \mathbf{I}_{v}^{t}}{1-Y_{1} \sin \left(Y_{2}\right)}\left(1-e \sin \left(m_{1}\right)-\sin \left(m_{1}\right)\right)+e \sin \left(m_{1}\right) \mathrm{B}_{v}^{t}+\sin \left(m_{1}\right) \mathrm{A}_{v}^{t} \\
& X_{v}^{t+1}=\frac{X_{v}^{t+1}}{1-Y_{1} \sin \left(Y_{2}\right)}\left(1-e \sin \left(m_{1}\right)-\sin \left(m_{1}\right)\right)-\frac{Y_{1} Y_{3} \sin \left(Y_{2}\right) \mathbf{I}_{v}^{t}}{1-Y_{1} \sin \left(Y_{2}\right)}\left(1-e \sin \left(m_{1}\right)-\sin \left(m_{1}\right)\right) \\
& +e \sin \left(m_{1}\right) \mathrm{B}_{v}^{t}+\sin \left(m_{1}\right) \mathrm{A}_{v}^{t} \\
& X_{v}^{t+1}-\frac{X_{v}^{t+1}}{1-Y_{1} \sin \left(Y_{2}\right)}\left(1-e \sin \left(m_{1}\right)-\sin \left(m_{1}\right)\right)=e \sin \left(m_{1}\right) \mathrm{B}_{v}^{t}+\sin \left(m_{1}\right) \mathrm{A}_{v}^{t}-\frac{Y_{1} Y_{3} \sin \left(Y_{2}\right) \mathrm{I}_{v}^{t}}{1-Y_{1} \sin \left(Y_{2}\right)} \\
& \left(1-e \sin \left(m_{1}\right)-\sin \left(m_{1}\right)\right) \\
& X_{v}^{t+1}\left(1-\frac{1-e \sin \left(m_{1}\right)-\sin \left(m_{1}\right)}{1-Y_{1} \sin \left(Y_{2}\right)}\right)=e \sin \left(m_{1}\right) \mathrm{B}_{v}^{t}+\sin \left(m_{1}\right) \mathrm{A}_{v}^{t}-\frac{Y_{1} Y_{3} \sin \left(Y_{2}\right) \mathrm{I}_{v}^{t}}{1-Y_{1} \sin \left(Y_{2}\right)} \\
& \left(1-e \sin \left(m_{1}\right)-\sin \left(m_{1}\right)\right) \\
& X_{v}^{t+1}\left(\frac{1-Y_{1} \sin \left(Y_{2}\right)-1+e \sin \left(m_{1}\right)-\sin \left(m_{1}\right)}{1-Y_{1} \sin \left(Y_{2}\right)}\right)=e \sin \left(m_{1}\right) \mathrm{B}_{v}^{t}+\sin \left(m_{1}\right) \mathrm{A}_{v}^{t}-\frac{Y_{1} Y_{3} \sin \left(Y_{2}\right) \mathbf{I}_{v}^{t}}{1-Y_{1} \sin \left(Y_{2}\right)} \\
& \left(1-e \sin \left(m_{1}\right)-\sin \left(m_{1}\right)\right)
\end{aligned}
$$




$$
\begin{aligned}
& X_{v}^{t+1}\left(\frac{e \sin \left(m_{1}\right)+\sin \left(m_{1}\right)-Y_{1} \sin \left(Y_{2}\right)}{1-Y_{1} \sin \left(Y_{2}\right)}\right)=e \sin \left(m_{1}\right) \mathrm{B}_{v}^{t}+\sin \left(m_{1}\right) \mathrm{A}_{v}^{t}-\frac{Y_{1} Y_{3} \sin \left(Y_{2}\right) \mathbf{I}_{v}^{t}}{1-Y_{1} \sin \left(Y_{2}\right)} \\
& \left(1-e \sin \left(m_{1}\right)-\sin \left(m_{1}\right)\right) \\
& X_{v}^{t+1}=\left(\frac{1-Y_{1} \sin \left(Y_{2}\right)}{e \sin \left(m_{1}\right)+\sin \left(m_{1}\right)-Y_{1} \sin \left(Y_{2}\right)}\right)\left\{e \sin \left(m_{1}\right) \mathrm{B}_{v}^{t}+\sin \left(m_{1}\right) \mathrm{A}_{v}^{t}-\frac{Y_{1} Y_{3} \sin \left(Y_{2}\right) I_{v}^{t}}{1-Y_{1} \sin \left(Y_{2}\right)}\left(1-e \sin \left(m_{1}\right)-\sin \left(m_{1}\right)\right)\right\}
\end{aligned}
$$

where, $Y_{1}=x-o \frac{x}{Z}$. Here, the term $o$ denotes current iteration, $Z$ refer to the maximal iterations, $x$ represents the constant.

$$
\mathrm{A}_{v}^{t}=\frac{X_{\phi}+X_{\gamma}+X_{\beta}}{3}
$$

where, $X_{\varnothing}, X_{\gamma}, X_{\beta}$ denotes the three best solutions, and $B_{v}^{t}$ represents the best solution. $e^{t+1}=\alpha e^{t} ; 0<\alpha<1$ the value of $m_{1}, m_{2}$ ranging from 0 to 1 .

when $m_{2}>0.5$,

$$
X_{v}^{t+1}=\left(\frac{1-Y_{1} \sin \left(Y_{2}\right)}{e \cos \left(m_{1}\right)+\cos \left(m_{1}\right)-Y_{1} \sin \left(Y_{2}\right)}\right)\left\{e \cos \left(m_{1}\right) \mathrm{B}_{v}^{t}+\cos \left(m_{1}\right) \mathrm{A}_{v}^{t}-\frac{Y_{1} Y_{3} \sin \left(Y_{2}\right) \mathrm{I}_{v}^{t}}{1-Y_{1} \sin \left(Y_{2}\right)}\left(1-e \cos \left(m_{1}\right)-\cos \left(m_{1}\right)\right)\right\}
$$

The term $B_{v}^{t}$ refers to the ability of SSCA for better solution. The above equation specifies the updated equation of the proposed SSCA, which in turn used to perform cross layer resource allocation effectively.

Step 4: Compute the feasibility: After evaluating the updated position, the objective function of each solution is computed and the solution yielding maximum fitness is considered as best solution.

Step 5: Termination: The steps from 2 to 4 are repeated until the specified iteration met or the best solution is obtained. Algorithm 1 represents the pseudo code of developed SSCA algorithm.

Algorithm 1. Pseudo code of the developed SSCA-based allocation

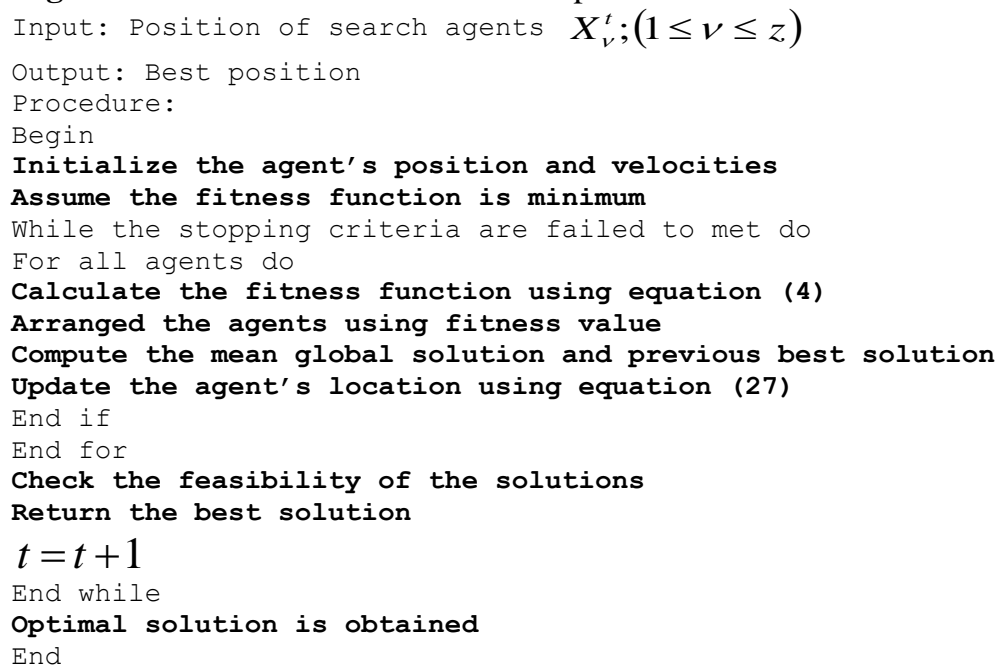

\section{RESULTS AND DISCUSSIONS}

The analysis of cross-layer resource allocationusing the proposed SSCA-based allocation is elaborated in this section to prove the effectiveness of the proposed model.

\subsection{Experimental set-up}

The proposed method is executed in 4GB RAM, Windows 8 OS with Intel core i-3 processor and the implementation is done in MATLAB.

\subsection{Evaluation metrics}

The performance revealed by the developed approach is evaluated using energy, throughput and the fairness. 


\subsection{Comparative methods}

The performance increased by the developed method is evaluated by comparing the proposed with existing methods, like waterfilling method [16], Security aware energy efficient allocation [19], distributed energy efficient allocation [24], respectively.

\subsubsection{Analysisbased on number of users}

Figure 2 shows the comparative of the developed SSCA-based allocation with respect to energy, throughput and the fairness with respect to users. Figure 2(a) represents the comparative analysis of the proposed SSCA-based allocation in terms of energy. When the count of user is 2, the energy obtained by the existing waterfilling method, Security aware energy efficient allocation, and distributed energy efficient allocation is $32943 \mathrm{~W}, 33157 \mathrm{~W}$, and $31168 \mathrm{~W}$, while the proposed SSCA-based allocation obtained better energy of 32173, respectively. When the count of user is 6 , the energy obtained by the proposed SSCA-based allocation is $38293 \mathrm{~W}$, while the percentage of improvement reported by the proposed method in comparison with the exiting waterfilling method, and security aware energy efficient allocation, distributed energy efficient allocation is $35678 \mathrm{~W}, 37920 \mathrm{~W}$, and $33999 \mathrm{~W}$, respectively.

Figure 2(b) represents the comparative analysis of the proposed SSCA-based allocation in terms of throughput by varying the users. When the count of user is 6 , the throughput obtained by the existing waterfilling method, Security aware energy efficient allocation, and distributed energy efficient allocationis $3.213 \mathrm{mbps}, 3.241 \mathrm{mbps}$, and $3.247 \mathrm{mbps}$, while the proposed SSCA-based allocation obtained better throughput of $3.292 \mathrm{mbps}$, respectively. When the count of user is 8 , the throughput obtained by the proposed SSCA-based allocation is $3.445 \mathrm{mbps}$, while the percentage of improvement reported by the proposed method in comparison with the exiting waterfilling method, Security aware energy efficient allocation, and distributed energy efficient allocation is $3.320 \mathrm{mbps}, 3.392 \mathrm{mbps}$, and $3.414 \mathrm{mbps}$, respectively. When the number of user is 10 , the throughput obtained by the proposed SSCA-based allocation is $3.608 \mathrm{mbps}$, while the percentage of improvement reported by the proposed method in comparison with the exiting waterfilling method, Security aware energy efficient allocation, and distributed energy efficient allocation is $3.396 \mathrm{mbps}, 3.540 \mathrm{mbps}$, and $3.577 \mathrm{mbps}$, respectively.

Figure 2(c) represents the comparative analysis of the proposed SSCA-based allocation in terms of fairness by varying the users. When the count of user is 6 , the fairness obtained by the existing waterfilling method, Security aware energy efficient allocation, and distributed energy efficient allocation is $0.760,0.770$, and 0.788 , while the proposed SSCA-based allocation obtained better fairness of 0.849 , respectively. When the count of user is 8 , the fairness obtained by the existing waterfilling method, Security aware energy efficient allocation, and distributed energy efficient allocation is $0.776,0.855$, and 0.860 , while the proposed SSCAbased allocation obtained better fairness of 0.861, respectively. When the count of user is 10 , the fairness obtained by the existing waterfilling method, security aware energy efficient allocation, and distributed energy efficient allocation is $0.805,0.863$, and 0.866 , while the proposed SSCA-based allocation obtained better fairness of 0.868 , respectively.

\subsubsection{Analysis based on transmitters}

Figure 3 shows the comparative of the developed SSCA-based allocation in terms of energy, throughput and the fairness with respect to transmitters. Figure 3(a) represents the comparative analysis of the proposed SSCA-based allocation in terms of energy. When the count of transmitter is 5, the energy obtained by the existing waterfilling method, Security aware energy efficient allocation, and distributed energy efficient allocation is $33336 \mathrm{~W}, 31604 \mathrm{~W}$, and $31420 \mathrm{~W}$, while the proposed SSCA-based allocation obtained better energy of $31709 \mathrm{~W}$, respectively. When the count of transmitter is 20 , the energy obtained by the proposed SSCA-based allocation is $39110 \mathrm{~W}$, while the percentage of improvement reported by the proposed method in comparison with the exiting waterfilling method, and Security aware energy efficient allocation, distributed energy efficient allocation is $35823 \mathrm{~W}, 38662 \mathrm{~W}$, and $39557 \mathrm{~W}$, respectively.

Figure 3(b) represents the comparative analysis of the proposed SSCA-based allocation in terms of throughput by varying the transmitters. When the count of transmitter is 10 , the throughput obtained by the existing waterfilling method, Security aware energy efficient allocation, and distributed energy efficient allocation is $3.019 \mathrm{mbps}, 3.039 \mathrm{mbps}$, and $3.134 \mathrm{mbps}$, while the proposed SSCA-based allocation obtained better throughput of $3.218 \mathrm{mbps}$, respectively. When the count of transmitter is 15 , the throughput obtained by the proposed SSCA-based allocation is $3.265 \mathrm{mbps}$, while the percentage of improvement reported by the proposed method in comparison with the exiting waterfilling method, Security aware energy efficient allocation, and distributed energy efficient allocation is $3.097 \mathrm{mbps}, 3.157 \mathrm{mbps}$, and $3.208 \mathrm{mbps}$, respectively. When the number of transmitter is 25 , the throughput obtained by the proposed SSCA-based allocation is $3.609 \mathrm{mbps}$, while the percentage of improvement reported by the proposed method in comparison with 
the exiting waterfilling method, Security aware energy efficient allocation, and distributed energy efficient allocation is $3.444 \mathrm{mbps}, 3.497 \mathrm{mbps}$, and $3.557 \mathrm{mbps}$, respectively.

Figure 3(c) represents the comparative analysis of the proposed SSCA-based allocation in terms of fairness by varying the transmitters. When the count of transmitter is 15 , the fairness obtained by the existing waterfilling method, Security aware energy efficient allocation, and distributed energy efficient allocation is 0.785 , 0.803 , and 0.809 , while the proposed SSCA-based allocation obtained better fairness of 0.811 . When the count of transmitter is 20 , the fairness obtained by the existing waterfilling method, Security aware energy efficient allocation, and distributed energy efficient allocation is $0.795,0.811$, and 0.812 , while the developed model obtained fairness of 0.818 , respectively. When the count of transmitter is 25 , the fairness obtained by the existing waterfilling method, Security aware energy efficient allocation, and distributed energy efficient allocation is $0.824,0.837$, and 0.855 , while the developed SSCA-based allocation obtained fairness of 0.868 .

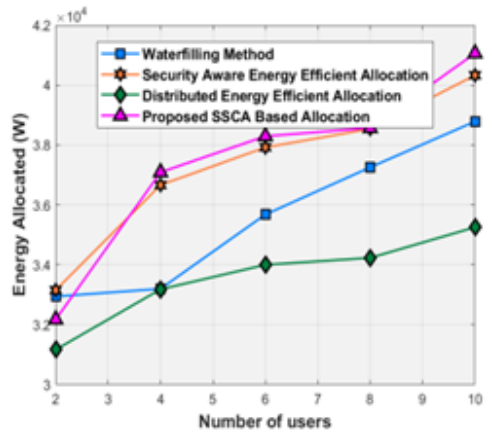

(a)

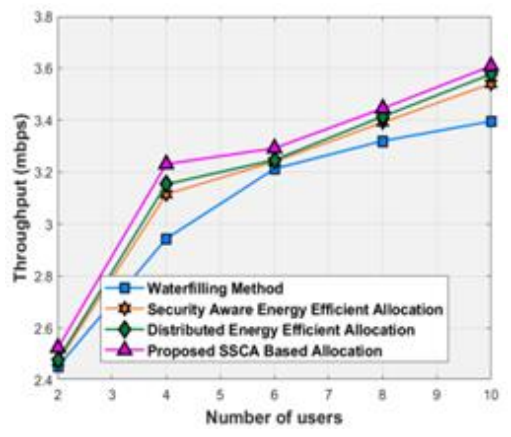

(b)

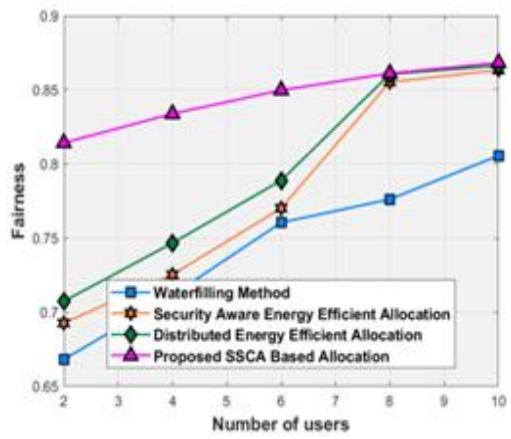

(c)

Figure 2. Comparative analysis of the developed method, a) energy, b) throughput, c) fairness

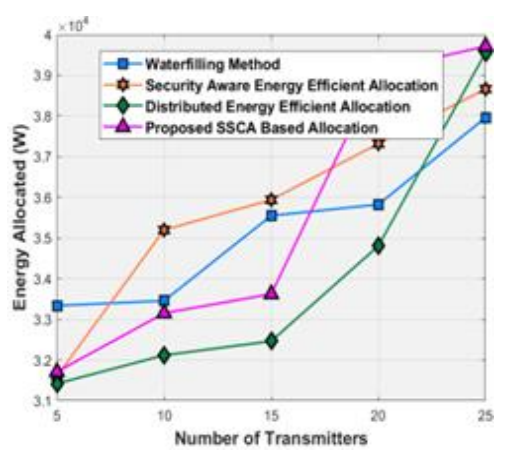

(a)

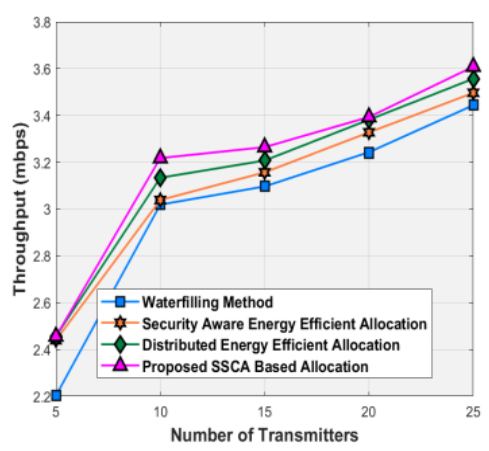

(b)

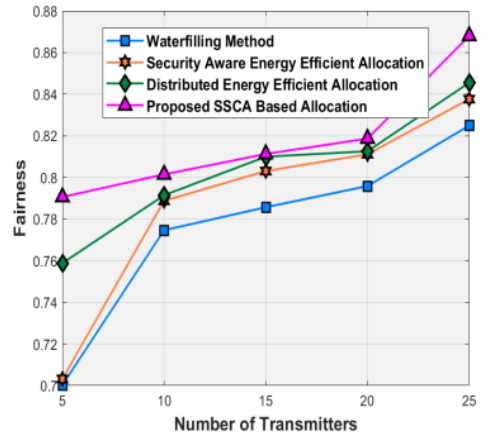

(c)

Figure 3. Comparative analysis of the developed method, a) energy, b) throughput, c) fairness

\subsubsection{Analysis based on subcarriers}

Figure 4 illustrates the comparative of the developed SSCA-based allocation with respect to energy, throughput and the fairness with respect to subcarriers. Figure 4(a) represents the comparative analysis of the proposed SSCA-based allocation in terms of energy. When the count of subcarrier is 16, the energy obtained by the existing waterfilling method, Security aware energy efficient allocation, and distributed energy efficient allocation is $21200 \mathrm{~W}, 18025 \mathrm{~W}$, and $18433 \mathrm{~W}$, while the proposed SSCA-based allocation obtained better energy of $22098 \mathrm{~W}$, respectively. When the count of subcarrier is 128 , the energy obtained by the proposed SSCA-based allocation is $134377 \mathrm{~W}$, while the percentage of improvement reported by the proposed method in comparison with the exiting waterfilling method, and Security aware energy efficient allocation, distributed energy efficient allocation is $129883 \mathrm{~W}, 125226 \mathrm{~W}$, and $127958 \mathrm{~W}$, respectively.

Figure 4(b) represents the comparative analysis of the proposed SSCA-based allocation in terms of throughput by varying the subcarriers. When the count of subcarrier is 32 , the throughput obtained by the existing waterfilling method, Security aware energy efficient allocation, and distributed energy efficient

Social-sine cosine algorithm-based cross layer resource allocation in wireless network (Praveena T.) 
allocation is $2.327 \mathrm{mbps}, 2.489 \mathrm{mbps}$, and $2.631 \mathrm{mbps}$, while the proposed SSCA-based allocation obtained better throughput of $2.700 \mathrm{mbps}$, respectively. When the count of subcarrier is 64 , the throughput obtained by the proposed SSCA-based allocation is $3.171 \mathrm{mbps}$, while the percentage of improvement reported by the proposed method in comparison with the exiting waterfilling method, Security aware energy efficient allocation, and distributed energy efficient allocation is $2.852 \mathrm{mbps}, 2.898 \mathrm{mbps}$, and $2.970 \mathrm{mbps}$, respectively. When the number of subcarrier is 256 , the throughput obtained by the proposed SSCA-based allocation is $3.703 \mathrm{mbps}$, while the percentage of improvement reported by the proposed method in comparison with the exiting waterfilling method, Security aware energy efficient allocation, and distributed energy efficient allocation is $3.486 \mathrm{mbps}, 3.502 \mathrm{mbps}$, and $3.685 \mathrm{mbps}$, respectively.

Figure 4(c) represents the comparative analysis of the proposed SSCA-based allocation in terms of fairness by varying the subcarriers. When the count of subcarrier is 32 , the fairness obtained by the existing waterfilling method, Security aware energy efficient allocation, and distributed energy efficient allocation is $0.735,0.739$, and 0.741 , while the proposed SSCA-based allocation obtained better fairness of 0.743 , respectively. When the count of subcarrier is 64 , the fairness obtained by the existing waterfilling method, Security aware energy efficient allocation, and distributed energy efficient allocation is $0.738,0.767$, and 0.776 , while the proposed SSCA-based allocation obtained better fairness of 0.809 , respectively. When the count of subcarrier is 256 , the fairness obtained by the existing waterfilling method, Security aware energy efficient allocation, and distributed energy efficient allocation is $0.794,0.820$, and 0.823 , while the proposed SSCA-based allocation obtained better fairness of 0.865 , respectively.

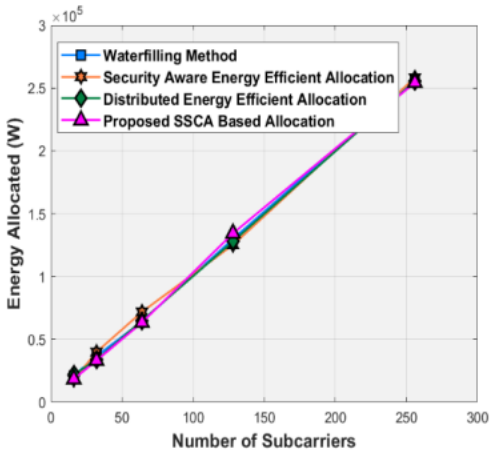

(a)

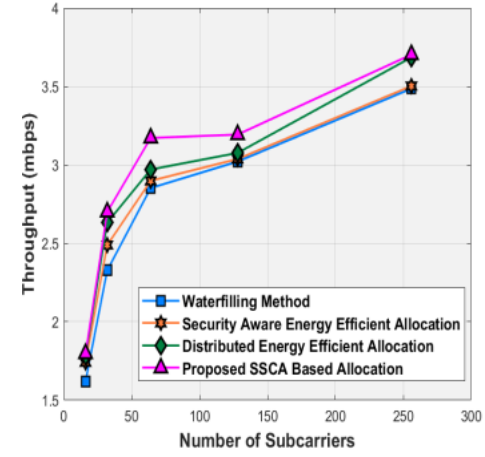

(b)

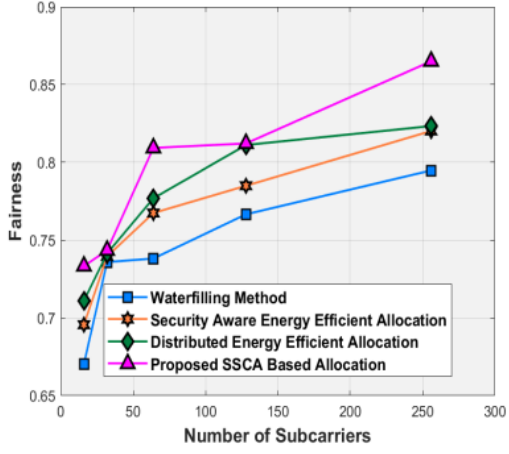

(c)

Figure 4. Comparative analysis of the developed method, a) energy, b) throughput, c) fairness

\subsection{Comparative discussion}

Table 1 depicts the comparative discussion of developed method by varying the users, transmitters, and the subcarriers. The energy obtained by the existing methods, like waterfilling method, Security aware energy efficient allocation, and distributed energy efficient allocation by varying the subcarriers is specified as $256553 \mathrm{~W}, 254324 \mathrm{~W}$, and $255357 \mathrm{~W}$, while the proposed SSCA-based allocation obtained better energy of $258213 \mathrm{~W}$, respectively. The throughput obtained by the existing methods, like waterfilling method, Security aware energy efficient allocation, and distributed energy efficient allocation by varying the subcarriers is specified as $3.486 \mathrm{mbps}, 3.502 \mathrm{mbps}$, and $3.685 \mathrm{mbps}$, while the proposed SSCA-based allocation obtained better throughput of $3.703 \mathrm{mbps}$, respectively.

Table 1. Comparative discussion

\begin{tabular}{clcccc}
\hline \multicolumn{2}{c}{ Metrics/Methods } & $\begin{array}{c}\text { Waterfilling } \\
\text { method }\end{array}$ & $\begin{array}{c}\text { Security aware energy } \\
\text { efficient allocation }\end{array}$ & $\begin{array}{c}\text { Distributed energy } \\
\text { efficient allocation }\end{array}$ & $\begin{array}{c}\text { Proposed SSCA- } \\
\text { based allocation }\end{array}$ \\
\hline Varying & Energy $(W)$ & 38784 & 40327 & 35253 & $\mathbf{4 1 0 5 9}$ \\
users & Throughput (mbps) & 3.396 & 3.540 & 3.577 & $\mathbf{3 . 6 0 8}$ \\
& Fairness & 0.805 & 0.863 & 0.866 & $\mathbf{0 . 8 6 8}$ \\
Varying & Energy $(W)$ & 37962 & 38662 & 39557 & $\mathbf{3 9 7 1 3}$ \\
transmitters & Throughput (mbps) & 3.444 & 3.497 & 3.557 & $\mathbf{3 . 6 0 9}$ \\
& Fairness & 0.824 & 0.837 & 255357 & $\mathbf{0 . 8 6 7}$ \\
Varying & Energy $($ W) & 256553 & 254324 & 3.685 & $\mathbf{2 5 8 2 1 3}$ \\
subcarriers & Throughput (mbps) & 3.486 & 3.502 & 0.823 & $\mathbf{3 . 7 0 3}$ \\
& Fairness & 0.794 & 0.820 & $\mathbf{0 . 8 6 5}$ \\
\hline
\end{tabular}

Int J Elec \& Comp Eng, Vol. 11, No. 1, February 2021 : 458 - 470 
It is clearly specified that the developed SSCA-based allocation better performance by varying the subcarriers, respectively. The fairness obtained by the existing methods, like waterfilling method, Security aware energy efficient allocation, and distributed energy efficient allocation by varying the users is specified as $0.805,0.863$, and 0.866 , while the proposed SSCA-based allocation obtained better fairness of 0.868 .

\section{CONCLUSION}

This paper presents the resource allocation approach using the proposed SSCA approach. The crosslayer optimization gets QSI and CSI from both MAC and physical layers. Based on energy and fairness, the cross-layer optimization-based resource allocation increases the sum rate of network. Then, the cross-layer optimization updates the decision using new input data with different channel conditions. Here, the resource allocation done based on proposed SSCA. The proposed SSCA is the combination of SSD and SCA. The proposed method and the fitness function, like fairness and energy enhance the overall system performance and allocating the resources effectively. The fairness function considered in this research are max-min, hard-fairness, mixed-bias, proportional fairness, and the maximum throughput. The performance of the resource allocation based on proposed model is evaluated based on energy, throughput, and the fairness. The performance of the resource allocation based on developed model is computed in terms of energy, throughput, and the fairness by varying users, transmitters, and the subcarriers. The developed model achieves the maximal energy of 258213, maximal throughput of 3.703, and the maximal fairness of 0.868 .

\section{REFERENCES}

[1] Khaled Ben Letaief, and Ying Jun (Angela) Zhang, "Dynamic multiuser resource allocation andadaptation for wireless systems," IEEE Wireless Communications, vol. 13, no. 4, pp. 38-47, 2006.

[2] Hai Jiang, Weihua Zhuang, and Xuemin (Sherman) Shen, "Cross-Layer Design for Resource Allocation in 3G Wireless Networks and Beyond," IEEE Communications Magazine, vol. 43, no. 12, pp. 120-126, 2005.

[3] L. Senthilkumar and M. Meenakshi, "Optimal Cross-Layer-Based Asymmetric Resource Allocation for Multidestination Relay Systems," IEEE transactions on wireless communications, vol. 17, no. 1, pp. 250-265, 2018.

[4] Pablo Ameigeiras, Juan J. Ramos-Munoz, Jorge Navarro-Ortiz, Preben Mogensen, and Juan M. Lopez-Soler, "QoE oriented cross-layer design of a resource allocation algorithmin beyond 3G systems," Computer Communications, vol. 33, no. 5, pp. 571-582, 2010.

[5] Antonio G. Marques, Member, Luis M. Lopez-Ramos, Georgios B. Giannakis, Javier Ramos, and A. J. Caamaño, "Optimal Cross-Layer Resource Allocation in Cellular Networks Using Channeland Queue-State Information," IEEE transactions on vehicular technology, vol. 61, no. 6, pp. 2789-2807, 2012.

[6] Guocong Song, and Ye Geoffrey Li, "Utility-Based Resource Allocation and Scheduling in OFDM-Based Wireless Broadband Networks," IEEE Communications magazine, vol. 43, no. 12, pp. 127-134, 2005.

[7] Xiaojun Lin, Ness B. Shroff, and R. Srikant, "A Tutorial on Cross-Layer Optimization in Wireless Networks," IEEE journal on selected areas in communications, vol. 24, no. 8, pp. 1452-1463, 2006.

[8] Irfan Ahmed, Amr Mohammed, and Hussein Alnuweiri, "On the fairness of resource allocation in wireless mesh networks: a survey," Wireless networks, vol. 19, no. 6, pp. 1451-1468, 2013.

[9] Jia Tang, and Xi Zhang,"Cross-Layer Resource Allocation Over Wireless Relay Networks for Quality of Service Provisioning," IEEE journal on selected areas in communications, vol. 25, no. 4, pp. 645-656, 2007.

[10] Vikas Kawadia, and P. R. Kumar, "A Cautionary perspective on cross-layer design," IEEE Wireless communications, vol. 12, no. 1, pp. 3-11, 2005.

[11] Eric Setton, Taesang Yoo, Xiaoqing Zhu, Andrea S. S. Goldsmith, and Bernd Girod, "Cross-layer design of ad hoc networks for real-time video streaming," IEEE Wireless Communications, vol. 12, no. 4, pp. 59-65, 2005.

[12] Fotis Foukalas, Vangelis Gazis, and Nancy Alonistioti, "Cross-layer design proposals for wireless mobile networks: a survey and taxonomy," IEEE communications Surveys \& Tutorials, vol. 10, no. 1, pp. 70-85, 2008.

[13] Bo Fu, Yang Xiao, Hongmei Julia Deng, and Hui Zeng, "A Survey of Cross-Layer Designs in Wireless Networks," IEEE communications surveys and tutorials, vol. 16, no. 1, pp. 110-126, 2013.

[14] Nessrine Chakchouk, and Bechir Hamdaoui, "Uplink Performance Characterization and Analysis of Two-Tier Femtocell Networks," IEEE transactions on vehicular technology, vol. 61, no. 9, pp. 4057-4068, 2012.

[15] Esmaeil Nik Maleki and Ghasem Mirjalily, "Cross layer resource allocation for faulttolerant topology control in wireless mesh networks based on genetic algorithm," EURASIP Journal on Wireless Communications and Networking, vol. 2019, pp. 1-24, 2019.

[16] Shu-Ming Tseng, Yung-Fang Chen, Po-Hsiang Chiu, and Hung-Chang Chi, "Jamming Resilient Cross-Layer Resource Allocation in Uplink HARQ-Based SIMO OFDMA Video Transmission Systems," IEEE Access, vol. 5, pp. 24908-24919, 2017.

[17] Young-Ho Jung, Qing Song, Kyung-Ho Kim, Pamela Cosman, and Laurence Milstein, "Cross-Layer Resource Allocation Using Video Slice Header Information for Wireless Transmission over LTE," IEEE Transactions on Circuits and Systems for Video Technology, vol. 28, no. 8, pp. 2024-2037, 2018.

[18] H. Kordbacheh, H. Dalili Oskouei, and N. Mokari, "Robust Cross-Layer Routing and Radio Resource Allocation in Massive Multiple antenna and OFDMA-based Wireless ad-hoc Networks," IEEE Access, vol. 7, pp. 36527-36539, 2019. 
[19] Lei Xu, Hong Xing, Arumugam Nallanathan, Yuwang Yang, and Tianyou Chai, "Security-Aware Cross-Layer Resource Allocation for Heterogeneous Wireless Networks," IEEE Transactions on communications, vol. 67, no. 2, pp. 1388-1399, 2019.

[20] Lei Xu and Weihua Zhuang, "Energy-Efficient Cross-layer Resource Allocation for Heterogeneous Wireless Access," IEEE Transactions on Wireless Communications, vol. 17, no. 7, pp. 4819-4829, 2018.

[21] Shu-Ming Tseng, and Yung-Fang Chen, "Average PSNR Optimized Cross Layer User Grouping and Resource Allocation for Uplink MU-MIMO OFDMA Video Communications," IEEE Access, vol. 6, pp. 50559-50571, 2018.

[22] Alaa Tharwat, and Thomas Gabel, "Parameters optimization of support vector machines for imbalanced data using social ski driver algorithm," Neural Computing and Applications, vol. 32, pp. 6925-6938, 2020.

[23] Seyedali Mirjalili, "SCA: A Sine Cosine Algorithm for Solving Optimization Problems," Knowledge-Based Systems, vol. 96, pp. 120-133, 2016.

[24] Irum Nosheen, Shoab A. Khan, Fatima Khalique, "A Mathematical Model for Cross Layer Protocol Optimizing Performance of Software Defined Radios in Tactical Networks," IEEE Access, vol. 7, pp. 20520-20530, 2019.

[25] Irfan Ahmed, Amr Mohamed, and Tarek Fouly, and Y. Charlie Hu, "On the Fairness of Frequency Domain Resource Allocation in Wireless Mesh Networks-A Survey," 2011 IEEE GCC Conference and Exhibition (GCC), Dubai, pp. 517-520, 2011.

\section{BIOGRAPHIES OF AUTHORS}

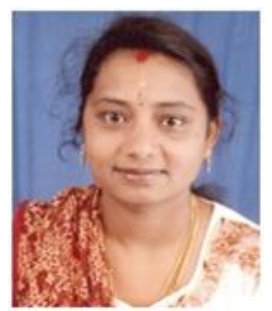

Praveena T., She is an Assistant Professor in Department of CSE R V College of Engineering. Her Specialization is Computer and Communication Networking, Algorithms, Wireless Networks and in core areas of computer science and Engineering. She has an experience of more than 15 years.

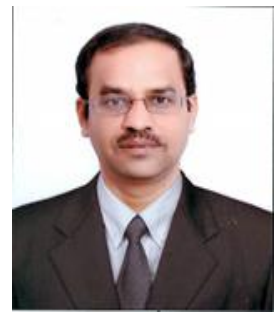

Dr. Nagaraja G. S., he is a Professor and Dean PG (CSE \& CNE) R V college of Engineering. His area of Specialization is Computer Networking, Network Management and core area of Computer Networking. He holds an experience of more than 24 years. 